\title{
Demons Meigs Syndrome: About Three Cases
}

\author{
Pr H. Benjelloun, Dr. M. Zerraa ${ }^{*}$, Pr. N. Zaghba, Pr. N. Yassine
}

Chu Ibn Rochd de Casablanca Rue des hôpitaux Casablanca, Marocoo

Introduction: Demons-Meigs syndrome associates a benign tumor of the ovary and serous effusions (pleural, peritoneal) recurrent most resorbable after removal of the primary tumor. Very rare, its physiopathology remains obscure. Through three observations and a review of the literature, we report the peculiarities of Demons meigs syndrome. Our Observations: We report three cases of patients aged 47 years, 51 years, and 63 years respectively. The reason for consultation was chest pain in all our patients. The clinical examination revealed a syndrome of unilateral fluid effusion in two cases, bilateral in one case, and physical signs of ascites in all three cases. Abdominalpelvic imaging demonstrated an ovarian-dependent abdomino-pelvic mass. The dosage of the CA125 tumor marker was very high in all our patients with an average of $741 \mathrm{IU} / \mathrm{ml}$. Surgical exploration revealed seroematic ascites with no suspect peritoneal lesions and a bulky ovarian fibrothecoma in all our patients. The operative sequences were simple, with a total and spontaneous draining of the effusions. Conclusion: Demons-Meigs syndrome is a benign pathology with good prognosis. The treatment is purely surgical. The excision of the ovarian tumor results in a drying of the pleural and peritoneal effusions.

Keywords: Demons- Meigs syndrome; épanchements; ovary CA125.

Copyright @ 2020: This is an open-access article distributed under the terms of the Creative Commons Attribution license which permits unrestricted use, distribution, and reproduction in any medium for non-commercial use (NonCommercial, or CC-BY-NC) provided the original author and source are credited.

\section{INTRODUCTION}

Demons-Meigs syndrome was first described in 1887 by Demons [1]. As early as 1866, Spiegel-berg showed out that this syndrome included a benign ovarian tumor associated with ascites and a recurrent hydrothorax [2]. Treatment is based on surgical resection of the ovarian tumor resulting into complete recovery and serous effusions's regression. The most commonly reported tumors are fibroids and thecomas [3]. This syndrome is often leading to fears of malignancy because of the existence of ascites and the significant elevation of CA125 [2]. Throughout three case reports and a review of the literature, we report the diagnosis, therapeutic and progressive features of Demons-Meigs syndrome.

\section{CASE REPORTS \\ Patient $n^{\circ} 1$}

It was a 51-year-old patient, with no specific medical background, presented to the pulmonology department for a right pleural effusion. Clinical examination revealed right fluid effusion syndrome, physical signs of ascites and a hard, mobile, abdominopelvic, palpable mass lateralized to the right, independent of the uterus with vaginal contact. The chest radiograph showed right pleural-like opacity. The pleural fluid matched to an exudate. The pleural needle biopsy did not help in the etiological diagnosis. Several evacuating pleural punctures were performed. The abdomino-pelvic ultrasound showed a heterogeneous, multi-lobed soft tissue mass of probably ovarian origin associated with significant ascites. Imagery (abdominal and thoracic ct) showed out compressive right hydrothorax, septate ascites and an ovarian tumor of $70 \mathrm{~mm}$ on $70 \mathrm{~mm}$. The CA125 level was very high at $412 \mathrm{IU} / \mathrm{ml}$ for a normal below 35IU / $\mathrm{ml}$. The patient underwent an exploratory laparotomy which underlined a smooth, hard tumor, developed at the expense of the right ovary, without lesions of peritoneal carcinosis or liver lesions. After the ecavuation of the serohematic ascistic fluid, a right ovarioctomy was performed. The pathology diagnosis was highlighting an ovarian fibrothecoma. There was full recovery after surgery, with regression of serous effusions. No recurrences were noted.

\section{Patient $\mathbf{n}^{\circ} \mathbf{2}$}

It was a 47-year-old patient, with no specific pathological history, referred to the pulmonology consultation for a predominantly right bilateral pleural effusion. The clinical examination revealed bilateral 
fluid effusion syndrome and abdominal distension. The chest radiograph showed out predominantly bilateral pleural type opacity on the right (Figure 1). The pleural fluid matched to an exudate. The pleural needle biopsy did not help in the etiological diagnosis. Multiple evacuating pleural punctures were performed. The abdominopelvic and endovaginal ultrasound highlighted a large hypoechoic abdominopelvic ovarian mass of regular contours. The abdominal computed tomography underlined an abdomino-pelvic mass of $22 \mathrm{~cm}$ (figure 2,3), polylobed and heterodense necrosis site pushing back the digestive structures and associated with an important peritoneal effusion and an important bilateral pleural effusion on the right side. The CA125 level showed a significant elevation to 771IU / mL. A laparotomy was performed; it highlighted a tumor of $30 \mathrm{~cm}$ in diameter, solid with cystic foci, firm, with a smooth surface at the expense of the left ovary (Figure 4), without peritoneal carcinosis signs. After ascites' evacuation, a left annexectomy completed with a complete hysterectomy was performed. The pathological examination confirmed the diagnosis of a $2 \mathrm{~kg}$ benign ovarian fibrothecoma. The surgery follow-up was simple with spontaneous effusions drying (Figure 5). No recurrences were noted.

\section{Patient $\mathbf{n}^{\circ} \mathbf{3}$}

A 63-years-old patient, with no specific medical history, was referred to the pulmonology department for left pleural effusion. Clinical examination revealed left fluid effusion syndrome, and physical signs of high abundant ascites. Chest X-ray showed a left pleural-like opacity. The pleural fluid matched to a transudate. The abdomino-pelvic ultrasound showed a heterogeneous, multilobed soft tissue-like mass, probably of ovarian origin, associated with significant ascites. Imagery (abdominal and thoracic CT scan) showed high abundant ascites and a pelvic solidocystic infiltrating mass of $150 \mathrm{~mm}$ by $140 \mathrm{~mm}$, lateralized more to the right than to the left, probably of bilateral ovarian origin. CA125 level was very high at $1042 \mathrm{IU} / \mathrm{mL}$. The patient underwent an exploratory laparotomy which noted a smooth, hard tumor, developed at the expense of the left ovary, with no signs of peritoneal carcinosis or liver damage. After ascitic serohematic fluid drainage, a right ovarectomy was performed. The pathology diagnosis was an ovarian fibrothecoma. The surgery follow-up was simple with regression of the serous effusions. No recurrences were noted.

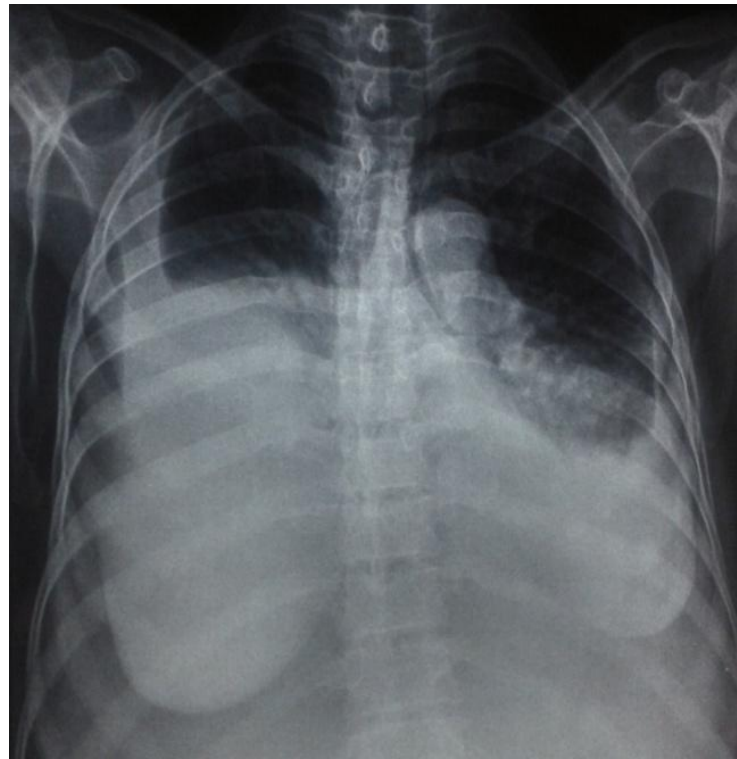

Fig-1: X-ray of the thorax showing a predominant bilateral pleural type opacity on the right

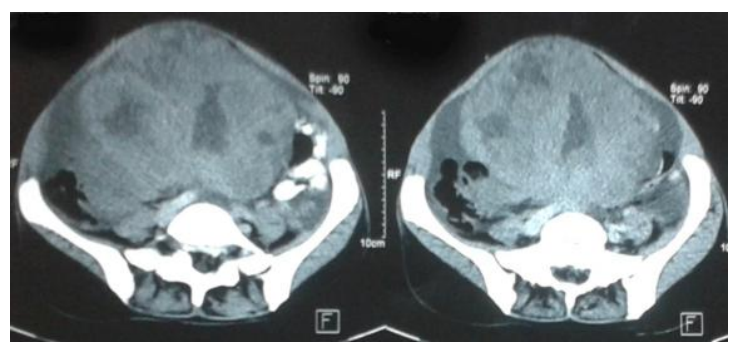

Fig-2: Pelvic CT scans showing a solidocystic right ovarian mass site of necrosis

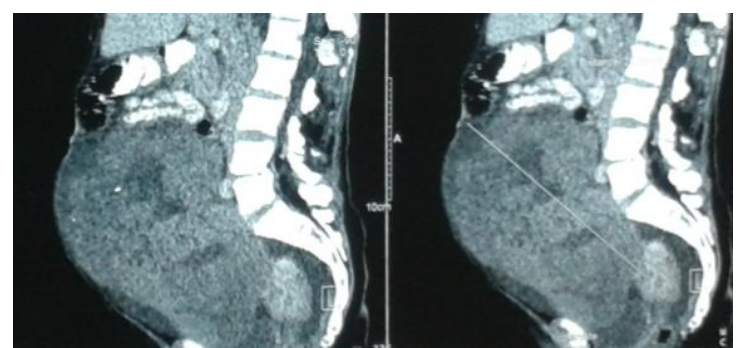

Fig-3: CT scans of pelvic reconstructions showing a solid ovarian mass that measured $22 \mathrm{~cm}$

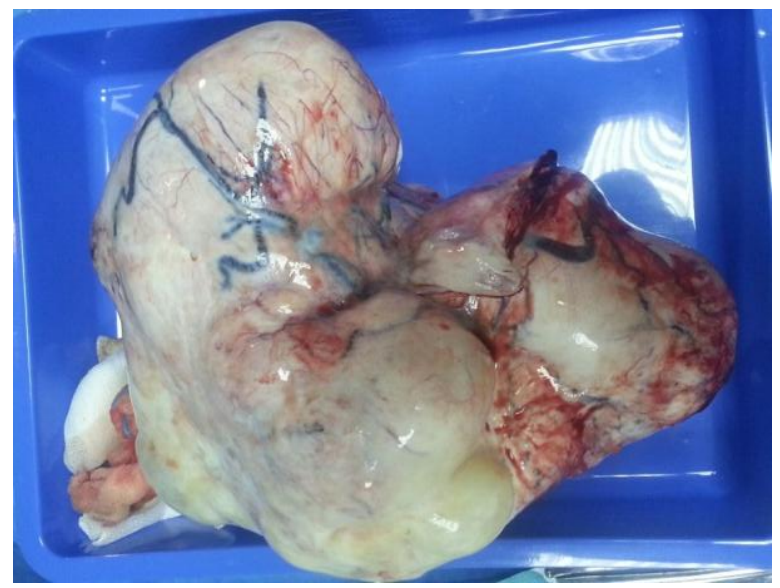

Fig-4: Surgical exploration showing a right ovary with a 2 kg tumor 


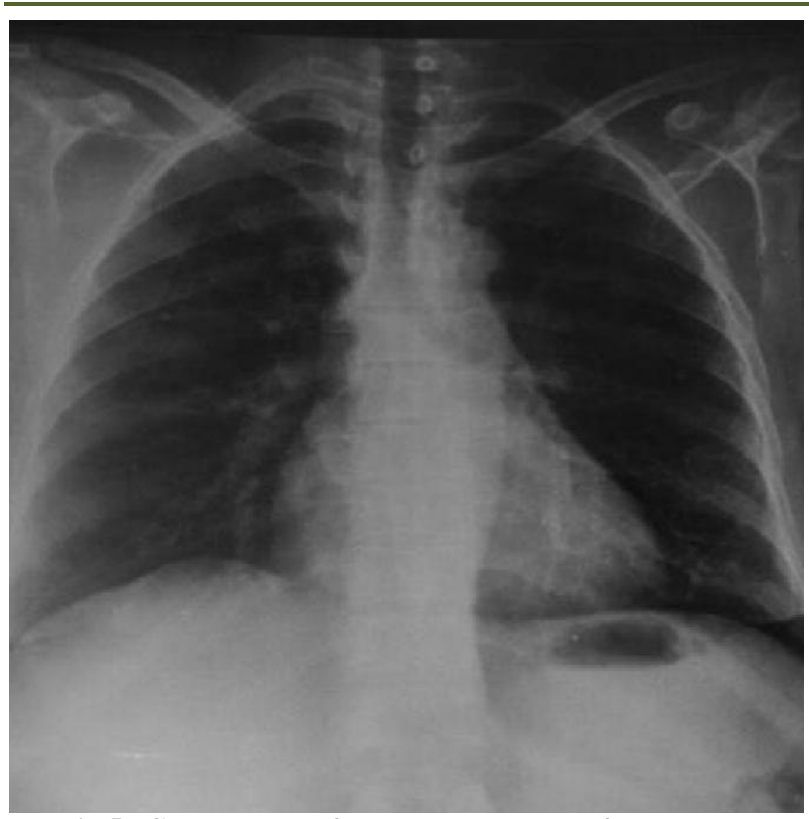

Fig-5: Chest $\mathrm{x}$-ray performed three months after surgery showing total dryness of bilateral pleurisy

\section{DISCUSSION}

In case of a thoracic and / or abdominal effusion in women, it is advised in practice, once tuberculosis, the mechanical causes are ruled out in our context, to think about a tumor of the reproductive system [4]. Thus the importance of ascites, especially right pleurisy, the ovarian tumor's volume, its weight, its benign character, the dramatic evolution towards the spontaneous effusions drying, and the patient's quick improvement after the tumor's removal[5] make these cases typical examples of Demons Meigs syndrome. If its pathogenesis remains most obscure, it is the anatomical, progressive and prognosis conditions added to the triad that have allowed this solitary entity to be particular syndrome [6]. The syndrome's definition is extended [7].

In this case some authors prefer to talk about pseudo-Demons-Meigs syndrome. it is frequent and generally associates ascites and / or pleural effusion with benign tumor, such as a para-ovarian fibroma [8, 9], a voluminous uterine leiomyoma [5], with the broad ligament [10] or even in the colon [8].

Several pathophysiological hypotheses have been Advanced concerning the effusions's genesis. For some, the appearance of ascites is explained by a partial obstruction of the venous return, linked to torsion of the pelvic tumor [11]. There would then be serous liquid transudation through the capsule. In fact, the tumors adhesions make torsion phenomena very rare $[1,11]$ and the ascites compositions and pleural effusion identical. Other authors have chosen ascites secretion by the tumor itself [11]. The ascites genesis is also explained by an increased pressure in the intratumoral lymphatics causing liquid leakage through the cubic epithelium [11, 12]. As far as pleural effusion is concerned, opinions are more consistent. The abdominal fluid crosses into the chest cavity through the diaphragmatic orifices (the characteristics of the fluids are identical). There is also peritoneal fluid crossing to the pleural cavity via the transdiaphragmatic lymphatic tract. This network is more developed on the right than on the left, hence the predominance of right pleural effusion.

Serous effusions generally correlated to citrus transudates; and can be exudative; these are rarely hemorrhagic effusions, as we have seen in our cases. The occurrence of effusion appears to correlate with tumor size and the presence of a myxoid component of the lesion. On the other hand, the effusions abundance is not related to the tumor volume [11].

In front of any unexplained pleurisy, a gynecological examination is essential in order to eliminate a malignant origin, especially in the presence of an ovarian mass with a high level of CA125.

In pelvic imaging, the ultrasound side of the ovarian mass depends on the age of the fibro-connective elements set up. Young tumors appear solid, hypoechoic and homogeneous. Old tumors are very absorptive, poorly defined and sometimes have calcifications. On CT, the most frequent image is that of a hypodense poly-lobed mass [7, 13], as we noted in our cases.

During this syndrome, it is classic to highlight an elevation of CA125 level, an antigenic tumor marker of the coelomic epithelium and its derivatives (peritoneum, pericardium and pleura) [14]. The high level of CA125 is not specific and should not suggest a malignant tumor. It has no diagnosis interest [4].

This has been illustrated in the literature, a study performed by Boufettal and Massoni found a CA125 level at 412IU / $\mathrm{ml}$ [3] and 752UI / ml respectively in the case of ovarian fibrothecoma [2]. In our cases, the preoperative levels are very high, especially in the $3 \mathrm{rd}$ case where this level reaches 1042IU / ml. The CA125 remains useful for postoperative monitoring [11].

However, the effusion's examination is essential in order to eliminate the malignant origin $[4$, $15,16]$.

The treatment of Demons-Meigs syndrome is surgical [3, 5, 17]. When the syndrome diagnosis is established pre-operatively and the patient is young, the treatment will be conservative to preserve her subsequent fertility. But doubtful diagnosis leads the surgeon to carry out a more or less wide excision. Extemporaneous histological examination can then be of great use in optimizing the extent of surgical excision by confirming the fibromatous and benign nature of the tumor [7]. In our second case, after evacuation of 
ascites, a Left annexectomy completed with a whole hysterectomy were performed. Surgical excision of the ovarian tumor generally allows spontaneous effusions drying [11], as we noted in our cases. A case of recurrence of Demons-Meigs syndrome was reported in the literature, occurring 30 years after the first treatment [11].

Our cases meet perfectly the criteria established by Meigs. These were benign fibrous ovarian tumors that were accompanied by ascites and hydrothorax. Surgical resection of the ovarian tumor led to spontaneous resolution of the effusions without recurrence for long.

\section{CONCLUSION}

Demons-Meigs syndrome consists mainly of a benign ovarian tumor, ascites and recurrent pleural effusion. This triad is not necessarily synonymous of malignancy and must be known by radiologists, pulmonologists and surgeons despite the fact that the serum CA125 level is often high. The definitive treatment for effusions is based on surgical removal of the ovarian tumor.

\section{Conflicts of interest}

The authors declare no conflict of interest

\section{REFERENCES}

1. Meigs JV, Cass JW. Fibroma of the ovary with ascites and hydrothorax: with a report of seven cases. American Journal of Obstetrics and Gynecology. 1937 Jan 1;33(2):249-67.

2. Massoni F, Carbillon L, Azria E, Uzan M. Demons-Meigs syndrome: apropos of 1 case. Gynecologie, obstetrique \& fertilite. 2001 Dec;29(12):905-7.

3. Boufettal H, Zaghba N, Morad S, Bakhatar A, Yassine N, Bahlaoui A, Noun M, Hermas S, Samouh N. Syndrome de Demons-Meigs: à propos d'une nouvelle observation et revue de la littérature. Revue de Pneumologie Clinique. 2011 Apr 1;67(2):121-3.

4. Brun GH. Syndromes et pseudosyndromes de Demons et Meigs aujourd'hui. Journal de gynécologie obstétrique et biologie de la reproduction. 2010 May 1;39(3):191-5.

5. Boufettal H, Elkerroumi M, Kamri Z, Mikou F, Ghazli M, Matar N. Syndrome de Demons-Meigs avec élévation importante du CA 125. Imagerie de la Femme. 2009 Jun 1;19(2):125-8.+Boufettal H, Elkerroumi M, Kamri Z, Mikou F, Ghazli M, Matar N. Syndrome de Demons-Meigs avec élévation importante du CA 125. Imagerie de la Femme. 2009 Jun 1;19(2):125-8.

6. Chammakhi-Jemli $\mathrm{CH}$, Chaaben I, Allani $\mathrm{H}$, Saddoud N, Moussa N, Sehili S, Daghfous MH. Volumineuse masse abdomino-pelvienne chez une femme âgée de 71 ans. Feuillets de radiologie. 2007 Dec 1;47(6):408-13.

7. Nemeth AJ, Patel SK. Meigs syndrome revisited. J Thorac Imaging. 2003;18:100-3.

8. Hanane B, Sanaa M, Nahid Z, Abdelaziz B, Najiba Y, Abdelkrim B. Pseudo-Meigs syndrome: à propos d'un cas. The Pan African medical journal. 2014;17.

9. Santangelo M, Battaglia M, Vescio G, Sammarco G, Gallelli G, Vetere A, Sommella L, Triggiani E. Meigs' syndrome: its clinical picture and treatment. Annali italiani di chirurgia. 2000;71(1):115-9.

10. Bretelle F, Portier M P, Boubli L, Houvenaeghel G. Syndrome de Demons-Meigs récidivé. À propos d'un cas. Ann Chir. 2000; 125: 269-72

11. Hirabayashi K, Graham J. Genesis of ascites in ovarian cancer. Am J Obstet Gynecol. 1970; 106: 492-7.

12. Bierman SM, Reuter KL, Hunter RE. Meigs'syndrome and ovarian fibrothecoma: CTfindings.J Comput Assist Tomog 1990;14:8334.

13. Le Bouedec G, Glowaczower E, de Latour M, Fondrinier E, Kauffmann P, Dauplat J. DemonsMeigs' syndrome. A case of thecoma and ovarian fibroma. J Gynecol Obstet Biol Reprod. 1992;21:651-4.

14. Dibonito L, Falconieri G, Colautti I, Bonifacio D, Dudine S. The peritoneal effusion: A retrospective study of cytopathologic diagnosis with autopsy confirmation. Acta Cytol. 1993; 37:483-8.

15. Oldrini G, Renard-Oldrini S, Vogin G, Marchal F, Grignon B, Henrot P. Ascite, épanchement pleural et lésion ovarienne. Presse Med. 2015 Oct;44(10):1076-8

16. Deffieux X, Thubert T, Huchon C, Demoulin G, Rivain AL, Faivre E, Trichot C. Complications des tumeurs ovariennes présumées bénignes. Journal de gynécologie obstétrique et biologie de la reproduction. 2013 Dec 1;42(8):816-32. 